\title{
A theory about the potential relationship between NAFLD and Blood cancers
}

\author{
A PHARMACOLOGICAL MEDIA REVIEW
}

Pardis Tabaee Damavandi | 04/05/2021

Subject to copyright law $2021 @$. The author is the only detainer of the copyright and intellectual property of the manuscript. The manuscript cannot be reproduced nor used without citation or due acknowledgement. 
Non-alcoholic fatty liver disease (NAFLD) is a condition that affects patients that suffer from obesity and excessive fatty acids leading to an inflammation of the liver. As we know this inflammation can become steatotic and thus chronic if the patient fails to reduce weight or exercise, leading to further complications from the protracted illness.

It has also previously been observed by many clinicians that there exists a correlation between ferritin levels, which are very high in patients who are about to develop Acute myeloid leukemia, or other blood cancers, and the incidence of blood cancers, nevertheless, an exaggerated amount of other studies are also controversial and confusing.

This short review wants to attempt to theorize a relationship between NAFLD and the incidence of blood cancers. To empirically support this, a metanalysis would be required.

- Firstly, NAFLD becomes chronic because weight loss appears very difficult for the patient to maintain, due to comorbidities, which make it "psychologically" hard to process. This conception of the liver failing, as well as the pain felt, by the patient, are barriers to the compliance in losing weight, changing diet, and exercising. A person suffering from NAFLD may just not be the sporty type, perhaps even due to arthritic disease and may not feel the strength, for the condition itself alone, to undertake long walks, or physical exercise, unless accompanied. On a psychological point of view, we could expect the patient being "enabled" into continuing as has always done, and the critical perspectives are often dismissed or ignored to enable further the patient into dismissing the condition. As all illnesses, an element of depression may result from too. Obviously a harsh approach is not going to work either, as it would just lead to patient withdrawal, nor is dishonesty though, as it would also lead to withdrawal, so there has to be a balance, when it comes to delivering information about liver disease, as if for whatever chronic conditions. Therefore, it is almost predictable, that NAFLD will be chronic before long.

Chronic disease thus means that we must expect inflammation somewhere in our organism. Iron noticeably binds the heme group therefore it is expected that it would get transported everywhere, as a bound component, especially in the blood compartment; as far as the unbound form is concerned, free iron is a metal ion in our organism, thus its trophism is like most metals, towards the bones and the blood. Therefore, elevated iron is expected to be a "chronic establishment" in our organism, meaning it leads to inflammation just by being present.

- We all know that chronic inflammation often results in desensitization, or an "adaptive mutation", which almost sounds like an oxymoron: "adaptive mutation", but mutations can be positive, when they are corrected by our repair enzymes, or negative, when they are not adjusted by our DNA repair enzymes. In the latter case, it is estimated that the failure in responding to that mutation appropriately can lead to the development of malignancies in the long term. 
- The positive feature about malignancy origin is, however, that it happens gradually and goes through stages, thus, it is predicted that with a ferritin count of less than $1000 \mu \mathrm{g} / \mathrm{L}$ the patient may not develop blood cancers, or certain mutations, and even though not all blood cancers have a good prognosis, it is reasonable to prevent them from occurring.

Some clinicians have proposed the approach of treating the fatty liver first and foremost, secondly it is logical to treat the iron overload from the liver damage to prevent the evolution into a worst case scenario. Treating iron overload is tricky as iron chelating agents can lead to side effects, such as iron deficiency anemia, with insomnia and all the potential negative aspects associated with the disorder, but dosage can be adjusted to suit the patient, even with drug interactions. To avoid this, the prophylaxis of Iron overload in NAFLD is better, through appropriate diagnostic tools, that involve not just MRI scans ${ }^{4}$, but ultrasound or biopsies.

In relation to the treatment of steatosis, newly patented Ivermectin and its analogues have been approved for NAFLD. Seemingly, the study shows that, aside of their parasitic activity, the disaccharide in this class of drugs is associated with a "liver cleansing" effect as opposed to their anti-parasitic activity displayed primarily by the Benzofuran ring.

Logically, diet and exercise would be the ideal approach to accompany the detoxification of the liver, but this proposal is rational in the short term, rather than in the long term, because the patient may not reach the goal of losing weight within that time period, and if the ferritin or iron levels do not drop, then pharmacological therapy should be considered.

\section{References}

1. Olcay, L ; Serteser, M; Kolay, M et al., The Impact of Iron Overload in Acute Leukemia: Chronic Inflammation, But Not the Presence of Nontransferrin Bound Iron is a Determinant of Oxidative Stress, Journal of Pediatric Hematology/Oncology: August 2017 Volume 39 - Issue 6 - p 425-439 doi: 10.1097/MPH.ooooooooooooo867

2. Marmur, J., Beshara, S., Eggertsen, G. et al. Hepcidin levels correlate to liver iron content, but not steatohepatitis, in non-alcoholic fatty liver disease. BMC Gastroenterol 18, 78 (2018). https://doi.org/10.1186/s12876-018-0804-o

3. Gillessen, A., Schmidt, H.HJ. Silymarin as Supportive Treatment in Liver Diseases: A Narrative Review. Adv Ther 37, 1279-1301 (2020). https://doi.org/10.1007/s12325-020-01251-y

4. Mobarra, N., Shanaki, M., Ehteram, H., et al. (2016). A Review on Iron Chelators in Treatment of Iron Overload Syndromes. International journal of hematology-oncology and stem cell research, 10(4), 239-247.

5. İdilman, İ. S., Akata, D., Özmen, M. N., et al. (2016). Different forms of iron accumulation in the liver on MRI. Diagnostic and interventional radiology (Ankara, Turkey), 22(1), 22-28. https://doi.org/10.5152/dir.2015.15094

\section{No conflicts of interest disclose.}

